\title{
A preclinical research platform to evaluate photosensitizers for transbronchial localization and phototherapy of lung cancer using an orthotopic mouse model
}

\author{
Tsukasa Ishiwata ${ }^{1,2}$, Takeshi Seki ${ }^{1,3}$, Alexander Gregor ${ }^{1}$, Masato Aragaki ${ }^{1}$, Yamato Motooka ${ }^{1}$, \\ Tomonari Kinoshita ${ }^{1}$, Terunaga Inage ${ }^{1}$, Nicholas Bernards ${ }^{1}$, Hideki Ujiie ${ }^{1}$, Zhenchian Chen ${ }^{1}$, \\ Andrew Effat ${ }^{1}$, Juan Chen ${ }^{4}$, Gang Zheng ${ }^{4,5,6,7}$, Koichiro Tatsumi ${ }^{2}$, Kazuhiro Yasufuku ${ }^{1,4,5,7}$ \\ ${ }^{1}$ Division of Thoracic Surgery, Toronto General Hospital, University Health Network, Toronto, Ontario, Canada; ${ }^{2}$ Department of Respirology, \\ Graduate School of Medicine, Chiba University, Chiba, Japan; ${ }^{3}$ Mechanical Engineering Course, Department of System Design Engineering, \\ Graduate School of Engineering Science, Akita University, Akita, Japan; ${ }^{4}$ Princess Margaret Cancer Centre, University Health Network/University \\ of Toronto, Toronto, Ontario, Canada; ${ }^{5}$ TECHNA Institute for the Advancement of Technology for Health, University Health Network, Toronto, \\ Ontario, Canada; ${ }^{6}$ Department of Medical Biophysics, University of Toronto, Toronto, Ontario, Canada; ${ }^{7}$ Institute of Biomaterials and Biomedical \\ Engineering, University of Toronto, Toronto, Ontario, Canada \\ Contributions: (I) Conception and design: T Ishiwata, T Seki, A Gregor, M Aragaki, Y Motooka, T Kinoshita, T Inage, N Bernards, H Ujiie, Z Chen, \\ A Effat; (II) Administrative support: J Chen, G Zheng, K Tatsumi, K Yasufuku; (III) Provision of study materials or patients: T Ishiwata, T Seki, M \\ Aragaki, Y Motooka; (IV) Collection and assembly of data: T Ishiwata, M Aragaki, Y Motooka; (V) Data analysis and interpretation: T Ishiwata, T \\ Seki, A Gregor, M Aragaki, Y Motooka, T Kinoshita, T Inage, N Bernards, H Ujiie, Z Chen, A Effat, J Chen; (VI) Manuscript writing: All authors; \\ (VII) Final approval of manuscript: All authors. \\ Correspondence to: Dr. Kazuhiro Yasufuku. Division of Thoracic Surgery, Toronto General Hospital, University of Toronto, 200 Elizabeth St, 9 N-957, \\ Toronto, Ontario, M5G 2C4, Canada. Email: kazuhiro.yasufuku@uhn.ca.
}

Background Establishing the efficacy of novel photosensitizers (PSs) for phototherapy of lung cancer
requires in vivo study prior to clinical evaluation. However, previously described animal models are not ideal
for assessing transbronchial approaches with such PSs.
Methods: An ultra-small parallel-type composite optical fiberscope (COF) with a 0.97 mm outer diameter
tip. The integration of illumination and laser irradiation fibers inside the COF allows simultaneous white-
light and fluorescence imaging, as well as real-time monitoring of tip position during laser phototherapy. An
orthotopic lung cancer mouse model was created with three human lung cancer cell lines transbronchially
inoculated into athymic nude mice. The COF was inserted transbronchially into a total of 15 mice for tumor
observation. For in vivo fluorescence imaging, an organic nanoparticle, porphysome, was used as a PS. Laser
excitation through the COF was performed at 50 mW using a 671 nm source.
Results: The overall success rate for creating orthotopic lung tumors was $71 \%$. Transbronchial white light
images were successfully captured by COF. Access to the left main bronchus was successful in $87 \%$ of mice
(13/15), the right main bronchus to the cranial lobe bronchus level in $100 \%(15 / 15)$, and to the right basal
trifurcation of the middle lobe, caudal lobe and accessory lobe in $93 \%$ (14/15). For transbronchial tumor
localization of orthotopic lung cancer tumors, PS-laden tumor with the strong signal was clearly contrasted
from the normal bronchial wall.

Conclusions: The ultra-small COF enabled reliable transbronchial access to orthotopic human lung cancer xenografts in vivo. This method could serve as a versatile preclinical research platform for PS evaluation in lung cancer, enabling transbronchial approaches in in vivo survival models inoculated with human lung cancer cells.

Keywords: Fluorescence imaging; photosensitizer; lung cancer; orthotopic lung cancer mouse model; bronchoscopy 
Submitted Jul 05, 2020. Accepted for publication Nov 04, 2020.

doi: $10.21037 /$ tlcr-20-813

View this article at: http://dx.doi.org/10.21037/tlcr-20-813

\section{Introduction}

Using photosensitizers (PSs) for tumor localization and phototherapy, such as photodynamic therapy (PDT) or photothermal therapy (PTT), has shown promising results in a variety of cancers including lung, bladder, brain, esophageal, cervical, and ovarian cancers (1-6). In lung cancer specifically, fluorescence imaging has been used for the detection of centrally located tumors $(1,7,8)$ and for assessment of visceral pleural invasion (9). Fluorescence imaging can be conducted for both the detection of cancer and for guiding phototherapy delivery. PDT has been performed clinically for lung cancer using porfimer sodium $\left(\right.$ Photofrin $^{\circledR}$ ) or talaporfin sodium (Laserphyrin ${ }^{\circledR}$ ); to date there has been no clinical trial of PTT for lung cancer. Phototherapy for lung cancer has several advantages over conventional treatment approaches. First, with many of the PSs established or under investigation, PS accumulation preferentially occurs in malignant tissue via the enhanced permeability and retention (EPR) effect $(10,11)$; active transport is an alternative mechanism that has been recently discussed in some nanoparticles (12). The activation of PSs to generate reactive oxygen species or heat (PDT and PTT, respectively) is limited only to the area irradiated by light at the appropriate wavelength. The combination of preferential PS accumulation and directed light application can reduce injury to otherwise normal tissue. Compared with thoracic surgery, phototherapy can be far less invasive; unlike radiotherapy, there are no total-dose limitations. Accordingly, lung cancer phototherapy can be repeatedly performed if necessary $(1,7)$. The lower laser energy generally required for PDT may also offer a better safety profile compared to other laser-based treatment modalities, such as Nd:YAG laser (13).

Despite their efficacy, a major barrier to wider adoption of PDT is phototoxicity. The persistence of PSs in the skin and the associated photosensitization requires prolonged light avoidance after PS administration. New PSs with a higher efficacy and less phototoxicity are needed; an ideal agent would rapidly clear from normal tissues and also yield reduced skin accumulation, reducing the need for light avoidance. Identifying a candidate PS worthy for clinical practice, whereby it is both efficacious and safe, requires in vivo laboratory investigation. Various preclinical animal models have been developed to evaluate PSs that preferentially accumulate in tumors (14). However, dedicated animal models for evaluation of tumor localization and phototherapy of lung cancer have not been fully developed. Previous research of PSs has extensively relied on mouse subcutaneous tumor models with human lung cancer cell line xenografts (15). This animal model has been extensively applied for oncology studies because of its low cost and reliability for creation, monitoring, and treatment delivery. However, it is welldocumented that the tumor biology of subcutaneous xenografts differs from clinical human tumors $(16,17)$. Microenvironment differences alter tissue structure and thus fail to recapitulate the clinical phenotype. To overcome these limitations, orthotopic tumor models have been developed (16). Orthotopic implantation is defined as tumor implantation in the site where the original carcinoma grew, creating a more appropriate microenvironment to evaluate normal tumor behavior. In mid-sized animals, a common orthotopic lung tumor model is the VX2 rabbit model. VX2 tumor-bearing models are able to form large primary tumors that closely imitate human lung cancer growth and metastasis (18). However, VX2 cells are not human lung cancer, instead being derived from a rabbit virus-induced papilloma (19). Conversely, immunodeficient mouse or rat orthotopic models rely on implantation of human lung cancer cells into the lung $(20,21)$. These smaller animals make studying transbronchial approaches more challenging. Transbronchial assessment for efficacy of PSs in orthotopic mouse/rat models with human lung cancer cells is not possible by conventional methods.

To overcome the size limitations of mice, we evaluated whether an ultra-small fiberscope could be used to create a preclinical platform for evaluating transbronchialdependent PS approaches in orthotopic human lung cancer xenograft models. We have previously reported on an ultrasmall parallel-type composite optical fiberscope (COF) with a $0.97-\mathrm{mm}$ outer diameter tip that was developed in collaboration with OK Fiber Technology (Kyoto, Japan). Its small size and tip flexibility enable insertion into ultrasmall airways (22). The integration of illumination and laser irradiation fibers inside the COF allows simultaneous 
white-light and fluorescence imaging, as well as real-time monitoring of tip position during laser phototherapy. A platform utilizing the ultra-small COF with orthotopic lung cancer mouse models would allow for performance of transbronchial imaging and phototherapy, evaluation of various human lung cancer subtypes, and prospective evaluation of phototherapy efficacy via survival analysis. We present the following article in accordance with the ARRIVE reporting checklist (available at http://dx. doi. org/10. 21037/tlcr-20-813).

\section{Methods}

\section{Parallel-type ultra-small COF}

The outer membrane of the COF's insertable section is made from polytetrafluoroethylene and coated with additional hydrophilic material to reduce frictional forces when in contact with the bronchial wall for smooth insertion. The minimum bend radius of the COF is approximately $15 \mathrm{~mm}$. This flexibility reduces excessive mechanical forces when pushed against the bronchial wall, reducing the risk for bronchial wall injury. The COF bundles a laser transmission fiber $(120 \mu \mathrm{m}$ diameter), approximately 300 illumination optical fibers and 9,000 imaging optical fibers (with a final resolution of 1 pixel per fiber). The COF was connected to a charge-coupled device camera system with an integrated notch filter which reduces glare from the direct reflection of laser light.

\section{Orthotopic mouse lung cancer model for transbronchial fluorescence imaging}

All animal studies were performed under an animal use protocol (AUP 4151) approved by the Animal Care Committee of the University Health Network, Toronto, Canada. Female athymic nude mice (NCr-Foxn1nu) were purchased from Taconic Farms Inc. Eight-to-12-weekold mice were kept (five per cage) in a climate-controlled room (temperature: $23-25{ }^{\circ} \mathrm{C}$, humidity: $50 \% \pm 5 \%$ ) with a 12-h light/dark cycle and fed with autoclaved chow diet and water. As we previously reported (21), athymic nude mice were anesthetized and inoculated with human lung cancer cells transbronchially. Human lung cancer cell lines included A549 (adenocarcinoma), NCI-H2170 (squamous cell carcinoma), and NCI-H82 (small cell lung cancer). A total of $100 \mu \mathrm{L}$ cell mixture [70\% cell suspension of $1.0 \times$
$10^{6}$ cells in phosphate-buffered saline, $30 \%$ extracellular matrix (Matrigel, Corning, NY, USA)] was inoculated. Starting at 3 weeks after inoculation, weekly computed tomography (CT) scans were performed to evaluate lung tumor growth. Mice with tumors more than $5 \mathrm{~mm}$ in diameter on axial CT images were selected for PS administration and fluorescence imaging.

\section{In vivo fluorescence imaging of lung cancer in mice}

The PS used in this study was porphysome, an organic nanoparticle whose multimodality properties (fluorescence, PDT, PTT) we have previously described (23). Porphysomes were synthesized as previously reported (24). For fluorescence imaging, laser excitation was performed at $50 \mathrm{~mW}$ using a 671-nm source (22). Fluorescence images were acquired through a 700-nm long-pass filter. A dose of $10 \mathrm{mg} / \mathrm{kg}$ porphysome was injected intravenously into the tail vein $48 \mathrm{~h}$ prior to transbronchial evaluation. On the procedure date, mice were anesthetized by isoflurane inhalation followed by an insertion protocol (Figure 1). The irradiation laser was active while the COF was advanced. After completing all procedures, mice were recovered from anesthesia and observed clinically for $1 \mathrm{~h}$. Autopsy was performed on all mice that died during the COF procedure.

\section{Ex vivo fluorescence imaging}

After euthanizing the mice, the lungs and trachea were resected en bloc for ex vivo evaluation of PS distribution using a spectral imaging system (Maestro, Cambridge Research and Instrumentation, MA, USA) with red filter (615-665 $\mathrm{nm}$ excitation, $700 \mathrm{~nm}$ long-pass emission, $500 \mathrm{~ms}$ exposure time). Transpleural evaluation of tumor fluorescence was performed using the COF at a distance of $10-15 \mathrm{~mm}$ from the tumor surface. The same laser power settings for in vivo fluorescence imaging were employed.

\section{Statistical analysis}

The success rate for creating orthotopic models was calculated for each cell line over two years' experience by three researchers ( $\mathrm{T}$ Ishiwata, M Aragaki, and Y Motooka; 2018 and 2019). The success rate of COF insertion into the orthotopic lung cancer mouse model was also calculated. All statistical analysis was carried out using GraphPad Prism 7 (GraphPad Software, San Diego, CA, USA). 

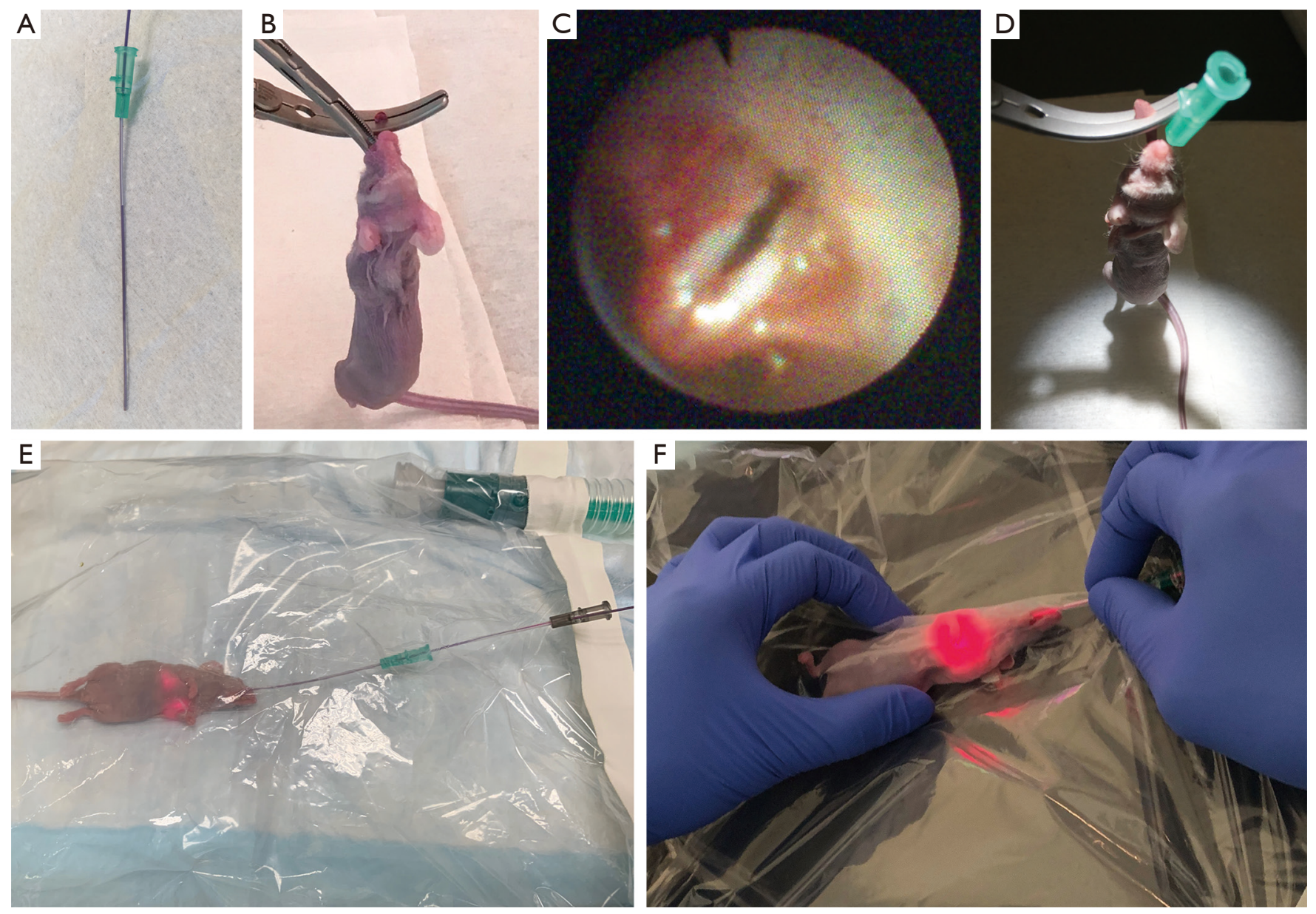

Figure 1 Transbronchial COF insertion into a mouse: the COF is fitted in an 18G intravenous catheter (Becton Dickinson, NJ, USA) to serve as a guide sheath (A). After the mouse is anesthetized, the tongue is extended out and held gently by an adjusted bulldog clip. A mosquito clamp is used to gently open the mouth to visualize the vocal cords (B). The COF is inserted into the mouse mouth under direct vision. After passing the vocal cords (C), the fiberscope is removed keeping the intravenous catheter within the trachea (D). The intubated mouse is placed in a prepared plastic bag filled with $3 \%$ isoflurane/97\% oxygen on a heating pad (E). The plastic bag is punctured and the COF inserted into the $18 \mathrm{G}$ endotracheal sheath. The $18 \mathrm{G}$ sheath is then removed from the trachea to maximize space around the COF during tumor localization. The direction of COF advancement can be altered through gentle manipulation of the mouse thorax position (F). $\mathrm{COF}$, composite optical fiberscope.

\section{Results}

\section{COF insertion in orthotopic lung cancer mouse models}

The overall success rate for creating orthotopic lung tumors was $71 \%$ [62/87]; this differed slightly between cell types (Figure S1). To assess the feasibility of transbronchial insertion, the COF was inserted into 15 orthotopic lung cancer mice with an average weight of $23.3 \mathrm{~g}$ (range, 19.2-26.2 g). White light observation of the bronchial lumen is shown in Figure $2 A$ and Video 1. Access to the left main bronchus was successful in $87 \%$ of mice [13/15], the right main bronchus to the cranial lobe bronchus level in $100 \%[15 / 15]$, and to the right basal trifurcation of the middle lobe, caudal lobe and accessory lobe in 93\% [14/15] (Figure 2B). Thirteen mice survived a continuous $15 \mathrm{~min}$ insertion of the COF for localizing the tumor. Two mice died during the procedure, with dyspnea first noted at around $10 \mathrm{~min}$. Autopsy of the two mice revealed no obvious airway injury or edema. A representative case of COF insertion and white-light observation of an orthotopic 
A
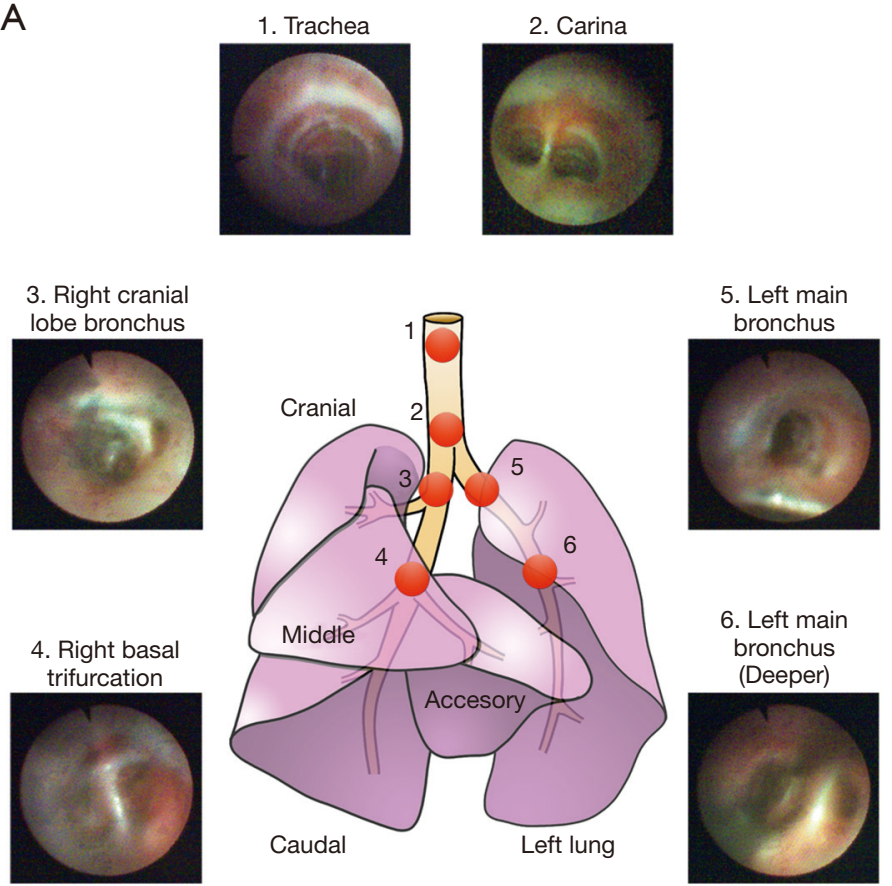

B

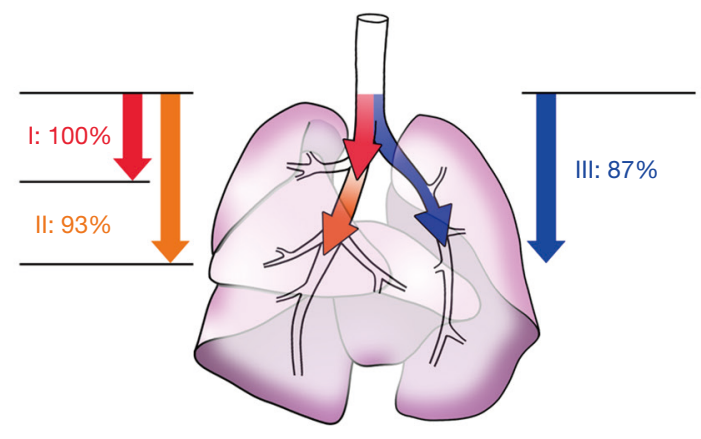

C
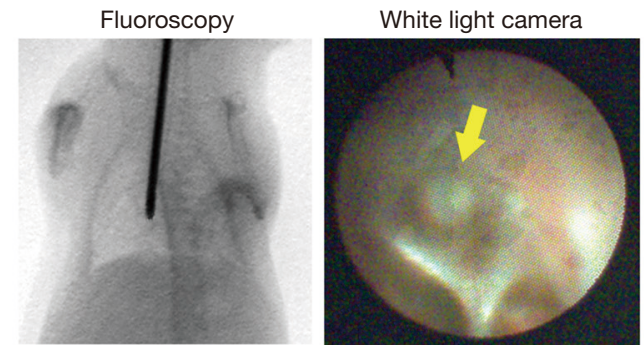

Figure 2 Bronchoscopy in the orthotopic lung cancer mouse model using the COF. (A) Anatomy of the mouse lung and corresponding white light camera images of the trachea and bronchi. The mouse right lung contains four lobes: cranial, middle, caudal, and accessory. The left lung is a single lobe. The COF can approach the entrance of each lobe bronchus. (B) The success rate of COF insertion was generally excellent but decreased as the COF was advanced more distally. All insertions were successful to the right cranial lobe bronchus level (zone I, red), 93\% [14/15] to the trifurcation of the basal bronchi (zone II, orange) and 87\% [13/15] from the carina to the left main bronchus (zone III, blue). (C) Representative fluoroscopic and white light images of the COF when approaching an orthotopic NCI-H82 lung cancer tumor made in the caudal lobe (yellow arrow). COF, composite optical fiberscope.

lung cancer tumor is shown in Figure 2C.

\section{Transbronchial tumor localization of orthotopic lung cancer tumors}

Representative cases of tumor fluorescence detection are shown in Figure 3 and Video 2. The normal bronchial wall showed slight fluorescence during laser irradiation, but this was clearly contrasted from the strong signal seen in the tumor proper.

\section{Discussion}

This was the first study to establish a preclinical platform using mouse for in vivo evaluation of PSs for transbronchial tumor localization for human lung cancer cells. The ultra-small COF enabled reliable transbronchial access to orthotopic human lung cancer xenografts in vivo, despite the small size of the mouse hosts. In vivo tumor fluorescence was successfully detected transbronchially. This preclinical animal model platform can readily be employed for evaluating other PSs for lung cancer diagnosis and treatment; the COF can be modified to irradiate and capture light at a variety of wavelengths.

From a safety perspective, transbronchial approaches for fluorescence imaging or phototherapy are favored in the clinical setting due to a reduced risk for complications including major hemorrhage or air embolism $(25,26)$. The accumulation of PS in adjacent normal tissues (e.g., bronchial mucosa) must be considered for transbronchial tumor localization, as this may reduce the ability of the clinician to delineate the tumor from normal background and thus compromise accurate phototherapy light delivery. Parameters that may be critical for optimal contrast, including PS concentration and the drug injection-light delivery interval, can be readily modified in our platform. 


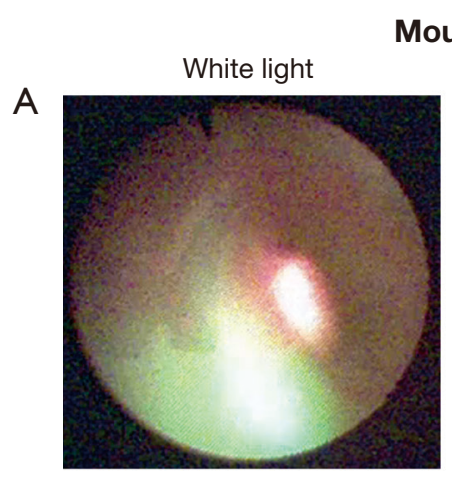

Tumor

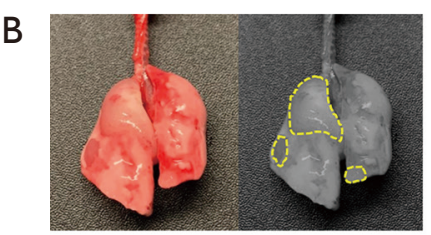

White light

C

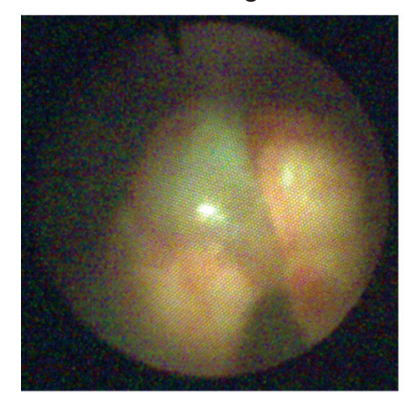

Mouse 1

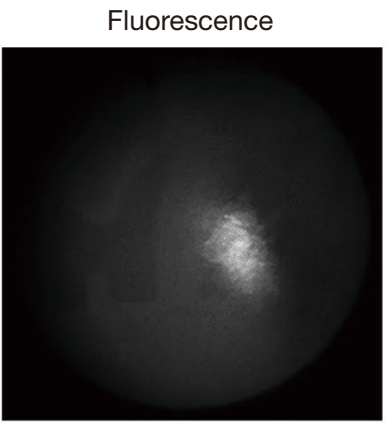

Ctrl

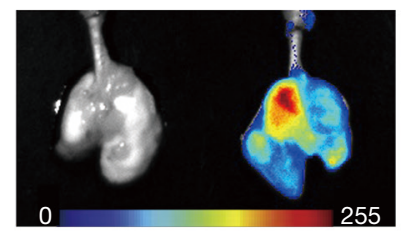

Fluorescence

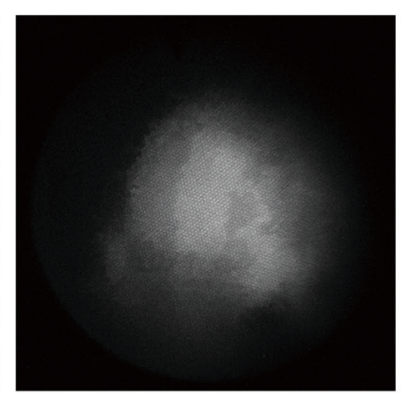

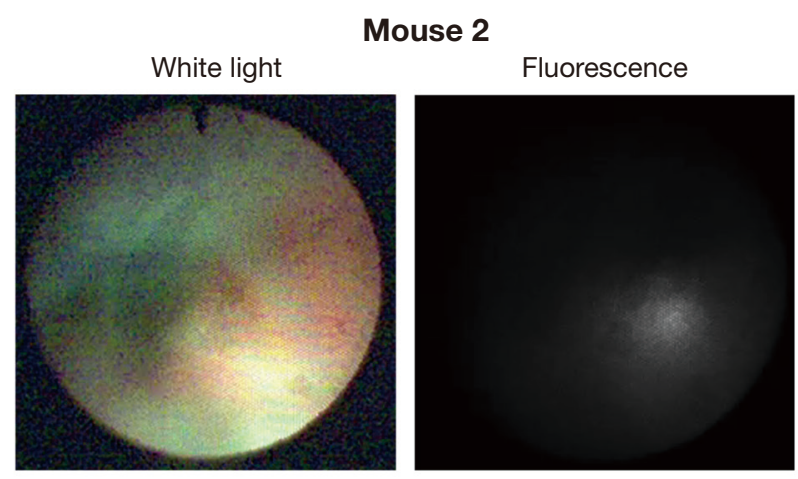

Tumor

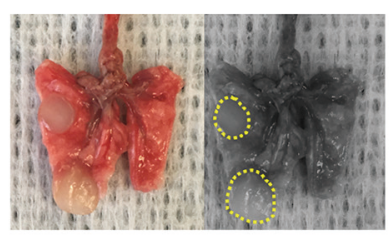

White light

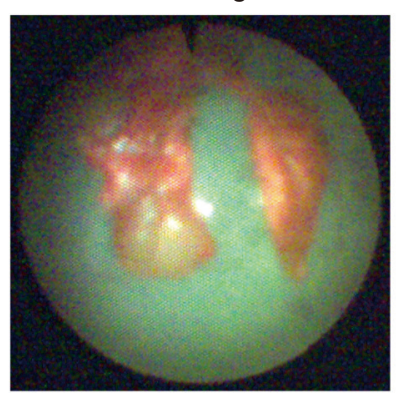

Ctrl

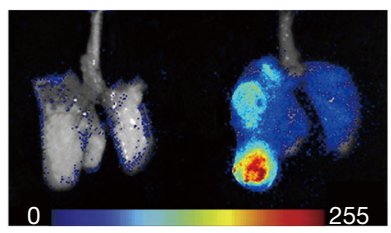

Fluorescence

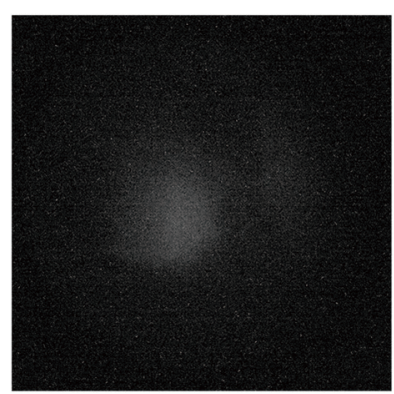

Figure 3 Representative mouse cases for fluorescence detection of orthotopic lung tumors. (A) Transbronchial fluorescence imaging of porphysome-laden tumors. Mice were injected with $10 \mathrm{mg} / \mathrm{kg}$ porphysome $48 \mathrm{~h}$ prior to observation. The COF used a $671-\mathrm{nm}$ laser source at $50 \mathrm{~mW}$. Clear contrast from the adjacent normal bronchial mucosa can be appreciated. (B) Gross evaluation of the lungs revealed tumors consistent with the COF findings (yellow broken lines denote the tumor margins); ex vivo evaluation by a spectral imaging system (Maestro) confirmed increased accumulation of porphysome in neoplastic tissue. (C) Ex vivo transpleural fluorescence observation of the orthotopic tumors by the COF similarly reveals contrast between the neoplastic and adjacent normal tissue. The distance between the tip of the fiberscope and the tumors was $10-15 \mathrm{~mm}$. COF, composite optical fiberscope.

Our platform could enable longitudinal fluorescence imaging, greatly facilitating the identification of the optimal drug-light interval for imaging.

There are a limited number of reports about bronchoscopy in living mice to date. Figueiredo et al. acquired intratracheal images via a flexible fiberscope with a 10,000-pixel resolution (27), and Dames et al. demonstrated intratracheal observation and bronchoalveolar lavage using a miniaturized semirigid bronchoscope with a 3,000-pixel resolution (28). To our knowledge, our work is the first to report the success rate of bronchoscopic access to each major lobar branch. With the COF, even relatively peripheral parts of the bronchi (e.g., the right trifurcation of the basal bronchi) were accessible with a high success rate $(93 \%)$ because of its small size and flexibility. One of the advantages of the animal model in this study is its ability to simulate small nodules in narrow airways. There are few reports on using PSs for peripheral lung cancer via transbronchial approaches; rather, clinical practice employs fluorescence imaging and phototherapy for centrally located lung cancers almost exclusively. This is in part related to challenges accessing peripheral nodules. However, ongoing technological advances are making the peripheral bronchial tree more accessible. 
Robotic bronchoscopy, which enables access to peripheral lung lesions with high tip stability (29-31), has begun early phase clinical evaluation. The ability to reliably and safely access the peripheral lung may make phototherapy through robotic bronchoscopy a promising new approach for small peripheral cancers (32). The small airways of mice closely replicate the airway diameters seen in the peripheral human lung. The mouse trachea is less than $1.5 \mathrm{~mm}$ in internal diameter, which is equivalent to the small peripheral airways in the human lung. Furthermore, the cellular composition and organization of the mouse trachea is generally more similar to the distal human airway than the human central airways (33).

This study evaluated fluorescence imaging. A key next step will be performing phototherapy (PDT or PTT) following fluorescence-based tumor localization; the COF allows this to be done without exchanging the fiberscope. This model has some limitations, however. First, while subcutaneous tumors can be easily assessed by physical exam, lung orthotopic tumors rely on serial CT scans. Moreover, although the COF has excellent reach within the mouse bronchial tree, lung tumors can still be inadvertently inoculated in sites beyond its reach (see Figure $2 B$ ). Second, prolonged COF placement is not without risks; $13 \%$ [2/15] of mice died during the $15 \mathrm{~min}$ period of tumor localization and imaging. On autopsy, we found no obvious airway injuries. The cause of death remains unclear; possible causes include aspiration of secretions or laryngeal edema/spasm from mechanical stimulation. Distal airway injury by the tip of the fiberscope could also have been potentially missed on autopsy. Especially for the study of PDT, a survival model is essential due to the delay between laser irradiation and maximal treatment effect. This delay is due to the complex mechanisms of PDT, including direct anti-tumor effects (highly reactive oxygen species causing necrosis and/or apoptosis) (34) and indirect anti-tumor effects by vasculature disruption (35). Creating a model with a higher survival rate even with prolonged fiberscope insertion would be invaluable. One potential solution would be fractionated PDT irradiation, with pauses allowing for animal recovery. Further investigation will be required.

In conclusion, we have demonstrated that a novel ultra-small COF enabled intrabronchial observation and transbronchial fluorescence imaging of lung cancer in orthotopic mouse models with a high success rate. This animal model may serve as a preclinical platform for evaluating new PSs for transbronchial fluorescence imaging and phototherapy for lung cancer.

\section{Acknowledgments}

The authors are grateful to Dr. Lili Ding, Dr. Kiyoshi Oka and Ms. Sachiko Minakawa for their collaboration, and Ms. Judy McConnell for laboratory management.

Funding: This work was supported by The National Sanitarium Association, Innovative Research Program Grant 2018, and William Coco Chair in Surgical Innovation for Lung Cancer.

\section{Footnote}

Reporting Checklist: The authors have completed the ARRIVE reporting checklist. Available at http://dx.doi. org/10.21037/tlcr-20-813

Data Sharing Statement: Available at http://dx.doi. org/10.21037/tlcr-20-813

Conflicts of Interest: All authors have completed the ICMJE uniform disclosure form (available at http://dx.doi. org/10.21037/tlcr-20-813). MA reports grants from Japan Society for the Promotion of Science, during the conduct of the study; GZ has a patent related to porphysome (US9072774); KY reports grants from the National Sanitarium Association Innovative Research Program Grant (2018), and support from the William Coco Chair in Surgical Innovation for Lung Cancer, during the conduct of the study. The other authors have no conflicts of interest to declare.

Ethical Statement: The authors are accountable for all aspects of the work in ensuring that questions related to the accuracy or integrity of any part of the work are appropriately investigated and resolved. Experiments were performed under an approved protocol (AUP 4151) granted by the institutional Animal Care Committee of the University Health Network (Toronto, Canada), in compliance with University Health Network's institutional guidelines for the care and use of animals.

Open Access Statement: This is an Open Access article distributed in accordance with the Creative Commons Attribution-NonCommercial-NoDerivs 4.0 International License (CC BY-NC-ND 4.0), which permits the noncommercial replication and distribution of the article with the strict proviso that no changes or edits are made and the original work is properly cited (including links to both the 
formal publication through the relevant DOI and the license). See: https://creativecommons.org/licenses/by-nc-nd/4.0/.

\section{References}

1. Usuda J, Kato H, Okunaka T, et al. Photodynamic therapy (PDT) for lung cancers. J Thorac Oncol 2006;1:489-93.

2. Bochenek K, Aebisher D, Miedzybrodzka A, et al. Methods for bladder cancer diagnosis - The role of autofluorescence and photodynamic diagnosis. Photodiagnosis Photodyn Ther 2019;27:141-8.

3. Mahmoudi K, Garvey KL, Bouras A, et al. 5-aminolevulinic acid photodynamic therapy for the treatment of highgrade gliomas. J Neurooncol 2019;141:595-607.

4. Wu H, Minamide T, Yano T. Role of photodynamic therapy in the treatment of esophageal cancer. Dig Endosc 2019;31:508-16.

5. Meulemans J, Delaere P, Vander Poorten V. Photodynamic therapy in head and neck cancer: indications, outcomes, and future prospects. Curr Opin Otolaryngol Head Neck Surg 2019;27:136-41.

6. Matoba Y, Banno K, Kisu I, et al. Clinical application of photodynamic diagnosis and photodynamic therapy for gynecologic malignant diseases: A review. Photodiagnosis Photodyn Ther 2018;24:52-7.

7. Usuda J, Tsutsui H, Honda H, et al. Photodynamic therapy for lung cancers based on novel photodynamic diagnosis using talaporfin sodium (NPe6) and autofluorescence bronchoscopy. Lung Cancer 2007;58:317-23.

8. Kato H. Our experience with photodynamic diagnosis and photodynamic therapy for lung cancer. J Natl Compr Canc Netw 2012;10 Suppl 2:S3-8.

9. Kitada M, Ohsaki Y, Yasuda S, et al. Photodynamic diagnosis of visceral pleural invasion of lung cancer with a combination of 5-aminolevulinic acid and autofluorescence observation systems. Photodiagnosis Photodyn Ther 2017;20:10-5.

10. Matsumura Y, Maeda H. A new concept for macromolecular therapeutics in cancer chemotherapy: mechanism of tumoritropic accumulation of proteins and the antitumor agent smancs. Cancer Res 1986;46:6387-92.

11. Nakamura Y, Mochida A, Choyke PL, et al. Nanodrug Delivery: Is the Enhanced Permeability and Retention Effect Sufficient for Curing Cancer? Bioconjug Chem 2016;27:2225-38.

12. Sindhwani S, Syed AM, Ngai J, et al. The entry of nanoparticles into solid tumours. Nat Mater 2020;19:566-75.
13. Furukawa K, Okunaka T, Yamamoto H, et al. Effectiveness of Photodynamic Therapy and Nd-YAG Laser Treatment for Obstructed Tracheobronchial Malignancies. Diagn Ther Endosc 1999;5:161-6.

14. D'Hallewin MA, Berrahmoune S, Bezdetnaya L, et al. Orthotopic animal models for oncologic photodynamic therapy and photodiagnosis. Photodiagnosis Photodyn Ther 2007;4:230-6.

15. Rygaard J, Povlsen CO. Heterotransplantation of a human malignant tumour to "Nude" mice. Acta Pathol Microbiol Scand 1969;77:758-60.

16. Garber K. From human to mouse and back: 'tumorgraft' models surge in popularity. J Natl Cancer Inst 2009;101:6-8.

17. Talmadge JE, Singh RK, Fidler IJ, et al. Murine models to evaluate novel and conventional therapeutic strategies for cancer. Am J Pathol 2007;170:793-804.

18. Gregor A, Fujino K, Bernards N, et al. Rabbit VX2 lung tumor models can form early nodal metastases. World J Surg Oncol 2019;17:231.

19. Shope RE, Hurst EW. Infectious papillomatosis of rabbits: with a note on the histopathology. J Exp Med 1933;58:607-24.

20. Onn A, Isobe T, Itasaka S, et al. Development of an orthotopic model to study the biology and therapy of primary human lung cancer in nude mice. Clin Cancer Res 2003;9:5532-9.

21. Nakajima T, Anayama T, Matsuda Y, et al. Orthotopic lung cancer murine model by nonoperative transbronchial approach. Ann Thorac Surg 2014;97:1771-5.

22. Kinoshita T, Effat A, Gregor A, et al. A Novel Laser Fiberscope for Simultaneous Imaging and Phototherapy of Peripheral Lung Cancer. Chest 2019;156:571-8.

23. Lovell JF, Jin CS, Huynh E, et al. Porphysome nanovesicles generated by porphyrin bilayers for use as multimodal biophotonic contrast agents. Nat Mater 2011;10:324-32.

24. Jin CS, Lovell JF, Zheng G. One minute, sub-one-watt photothermal tumor ablation using porphysomes, intrinsic multifunctional nanovesicles. J Vis Exp 2013:e50536.

25. Wiener RS, Schwartz LM, Woloshin S, et al. Populationbased risk for complications after transthoracic needle lung biopsy of a pulmonary nodule: an analysis of discharge records. Ann Intern Med 2011;155:137-44.

26. Heerink WJ, de Bock GH, de Jonge GJ, et al. Complication rates of CT-guided transthoracic lung biopsy: meta-analysis. Eur Radiol 2017;27:138-48.

27. Figueiredo JL, Alencar H, Weissleder R, et al. Near 
infrared thoracoscopy of tumoral protease activity for improved detection of peripheral lung cancer. Int J Cancer 2006;118:2672-7.

28. Dames C, Akyuz L, Reppe K, et al. Miniaturized bronchoscopy enables unilateral investigation, application, and sampling in mice. Am J Respir Cell Mol Biol 2014;51:730-7.

29. Chen AC, Gillespie CT. Robotic Endoscopic Airway Challenge: REACH Assessment. Ann Thorac Surg 2018;106:293-7.

30. Chen AC, Pastis NJ, Machuzak MS, et al. Accuracy of a Robotic Endoscopic System in Cadaver Models with Simulated Tumor Targets: ACCESS Study. Respiration 2020;99:56-61.

31. Yarmus L, Akulian J, Wahidi M, et al. A Prospective Randomized Comparative Study of Three Guided Bronchoscopic Approaches for Investigating

Cite this article as: Ishiwata T, Seki T, Gregor A, Aragaki M, Motooka Y, Kinoshita T, Inage T, Bernards N, Ujiie H, Chen Z, Effat A, Chen J, Zheng G, Tatsumi K, Yasufuku K. A preclinical research platform to evaluate photosensitizers for transbronchial localization and phototherapy of lung cancer using an orthotopic mouse model. Transl Lung Cancer Res 2021;10(1):243-251. doi: 10.21037/tlcr-20-813
Pulmonary Nodules: The PRECISION-1 Study. Chest 2020;157:694-701.

32. Murgu SD. Robotic assisted-bronchoscopy: technical tips and lessons learned from the initial experience with sampling peripheral lung lesions. BMC Pulm Med 2019;19:89.

33. Rock JR, Randell SH, Hogan BL. Airway basal stem cells: a perspective on their roles in epithelial homeostasis and remodeling. Dis Model Mech 2010;3:545-56.

34. Oleinick NL, Morris RL, Belichenko I. The role of apoptosis in response to photodynamic therapy: what, where, why, and how. Photochem Photobiol Sci 2002;1:1-21.

35. Dolmans DE, Kadambi A, Hill JS, et al. Vascular accumulation of a novel photosensitizer, MV6401, causes selective thrombosis in tumor vessels after photodynamic therapy. Cancer Res 2002;62:2151-6. 

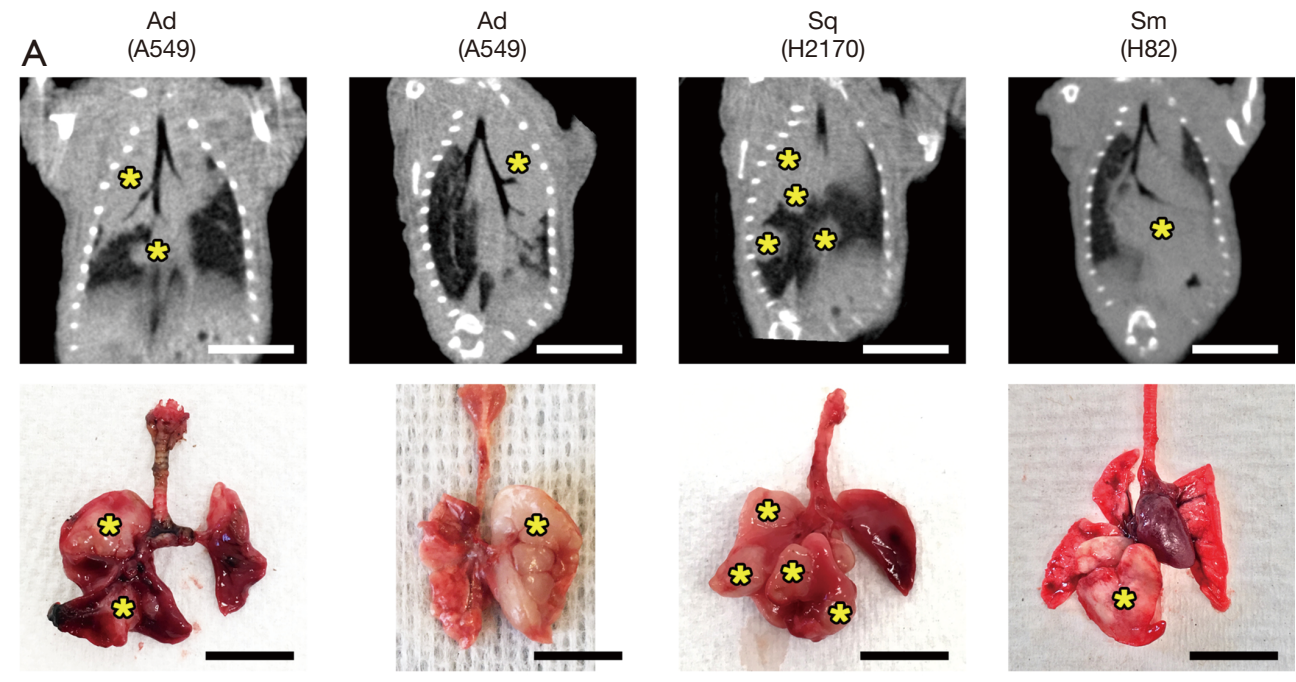

B

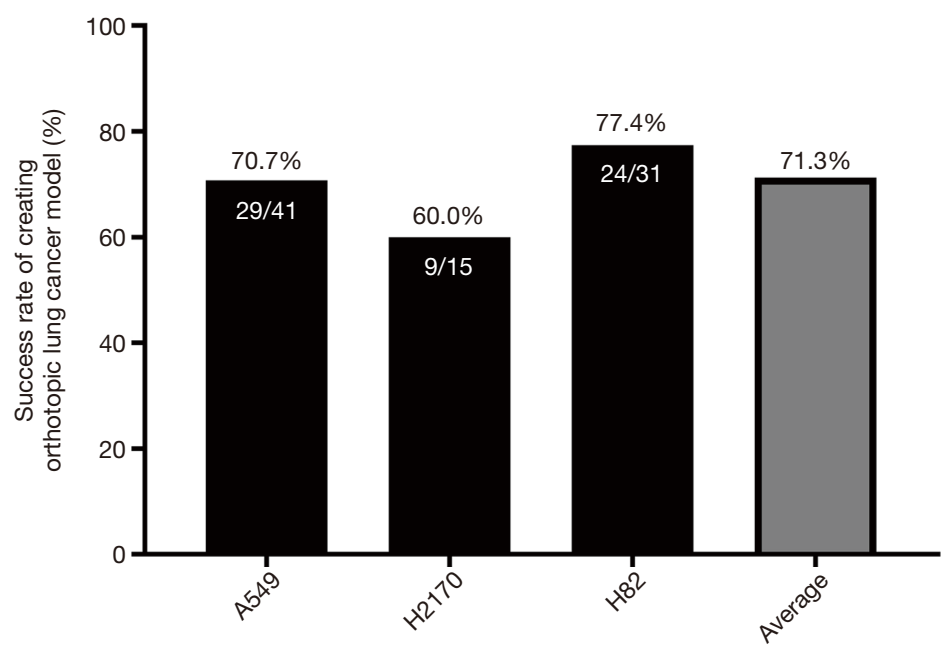

Figure S1 Mouse orthotopic lung cancer models. (A) Representative mouse orthotopic lung cancer models using human cell lines of adenocarcinoma (Ad, A549), squamous cell carcinoma (Sq, NCI-H2170) and small cell lung cancer (Sm, NCI-H82) are shown. Computed tomography multiplanar reconstructions (top row) and gross inspections of the lungs after sacrifice (bottom row) demonstrate successful tumor inoculation (yellow stars). Scale bar represents $10 \mathrm{~mm}$. (B) The success rate for creating orthotopic lung cancer tumors was calculated based on our group's experience from 2018 to 2019. Mice that immediately died during the cell-inoculation procedure are included in the denominator. Success did vary somewhat between cancer subtypes. 